\title{
Development of research on COVID-19 by the World Scientific Community in the first half of 2020
}

Daniel Tinôco ${ }^{1^{*}}$ and Suzana Borschiver ${ }^{2}$

DOl. 10.21931/RB/2020.05.04.23

Abstract: The World Scientific Community has carried out several studies on the novel coronavirus, responsible for the current COVID-19 pandemic. This study aimed to verify the development level and research evolution on COVID-19, summarizing experts' main trends in the first half of 2020. The most cited articles focused on understanding the disease, addressing aspects of its transmission, viral activity period, symptoms, health complications, risk factors, and the estimate of new cases. These papers also focused on the treatment/prevention and management of COVID-19. Several drugs and alternative treatments have been investigated, such as the convalescent plasma transfusion and stem cell transplantation, while an efficient vaccine is developed. Prevention and control measures, such as social isolation and immediate case identification, were also investigated. Therefore, the main COVID-19 trends were identified and classified in disease, treatment/prevention, and pandemic management, contributing to scientific understanding and future studies.

KeyWords: Novel coronavirus; SARS-CoV-2; COVID-19 scientific development; COVID-19 evolution; COVID-19 trends.

\section{Introduction}

At the end of December 2019, in Wuhan's city in China a novel coronavirus appeared, responsible for a severe acute respiratory syndrome, similar to the one in 2003, called SARS-CoV-2. A month later, the World Health Organization (WHO) declared that the coronavirus disease 2019 (COVID-19) outbreak consisted of a Public Health Emergency of International Importance, after H1N1 (2009), Poliomyelitis (polio) (2014), Ebola in West Africa (2014), Zika (2016), and Ebola in the Democratic Republic of Congo (2019). In March 2020, WHO declared the COVID-19 pandemic ${ }^{1}$.

In the first half of 2020 alone, the COVID-19 pandemic was marked by more than 11.6 million infected people, more than 500,000 deaths, and 216 affected countries worldwide, according to WHO. Until this moment, the USA and Brazil were the countries with most disease cases².

With the emergence and advancement of COVID-19, the world scientific community began a series of investigations and research to understand the behavior of the novel coronavirus in the human body, the consequences of the disease caused by it, and the most effective treatment for the combating of the COVID-19 pandemic. Furthermore, several studies have presented disease management guidelines or held discussions about implementing public health policies by the affected countries' governments ${ }^{3,4}$. Social isolation measures, rapid identification of suspected cases, and the tracking and followup of potential contacts between infected and non-infected were the main strategies suggested as efficient in preventing and controlling the COVID-194.

In this study, a prospection of scientific articles about COVID-19 was carried out, aiming to establish the scientific trajectory of the disease and identifying the development level of worldwide research on the novel coronavirus in recent months, specifically from February to June 2020. The selected papers were classified according to their approach, highlighting the transmission aspects, symptoms, risk factors, proposed treatments, clinical tests, and measures to prevent and control the disease.

\section{Scientific Studies}

China, the USA, and the U.K. were the countries with the most papers on COVID-19 cited by the scientific community. About 28\% of the scientific investigations were performed by Chinese research institutions and universities, followed by $16 \%$ from the USA and 11\% from the U.K. Several studies were conducted in hospitals by health professionals linked or not to the academy (Figure 1). Medical associations from different countries and national and international scientific research centers, such as the Centers for Disease Control and Prevention (USA) 5 have also developed research on the subject.

About $60 \%$ of the papers presented disease aspects, whose main nomenclatures were: COVID-19, SARS-CoV-2 (acute respiratory syndrome coronavirus 2), Novel coronavirus pneumonia, and Wuhan pneumonia. The novel coronavirus origin was attributed to two animals, the bat and the pangolin. For the bats, two types of viruses have been reported: batSL-CoVZC45 and bat-SL- CoVZXC216. For the pangolins, the Pangolin-CoV was identified ${ }^{7}$. About $23 \%$ of the papers corresponded to the disease's treatment and prevention investigations, and 17\% discussed COVID- 19 management (Figure 2). Many studies on the disease were due to the novelty of this virus, in which, despite the experience with Sars-CoV in 2003, little was known about the treatment and control forms. Therefore, in the first months, specialists investigated the disease mostly to understand it and control it.

\section{Scientific Trajectory}

The scientific trajectory revealed essential aspects for the understanding of the COVID-19. Approximately $70 \%$ of the papers discussed the disease in March 2020,50\% approached the treatment and prevention in February 2020, and 30\% suggested management measures of disease in May 2020 (Figure 3). The search for treatment before a complete understanding of the disease can be explained by the novelty and the rapid advance of COVID-19, which in February 2020 required immediate action to control the infection. With the disease development and the scientific research advancement, about $67 \%$ of the investigations in June 2020 focused on understanding the disease.

\footnotetext{
${ }^{1}$ Department of Biochemical Engineering, School of Chemistry, Federal University of Rio de Janeiro, Brazil.
}

${ }^{2}$ Department of Organic Processes, School of Chemistry, Federal University of Rio de Janeiro, Brazil. 


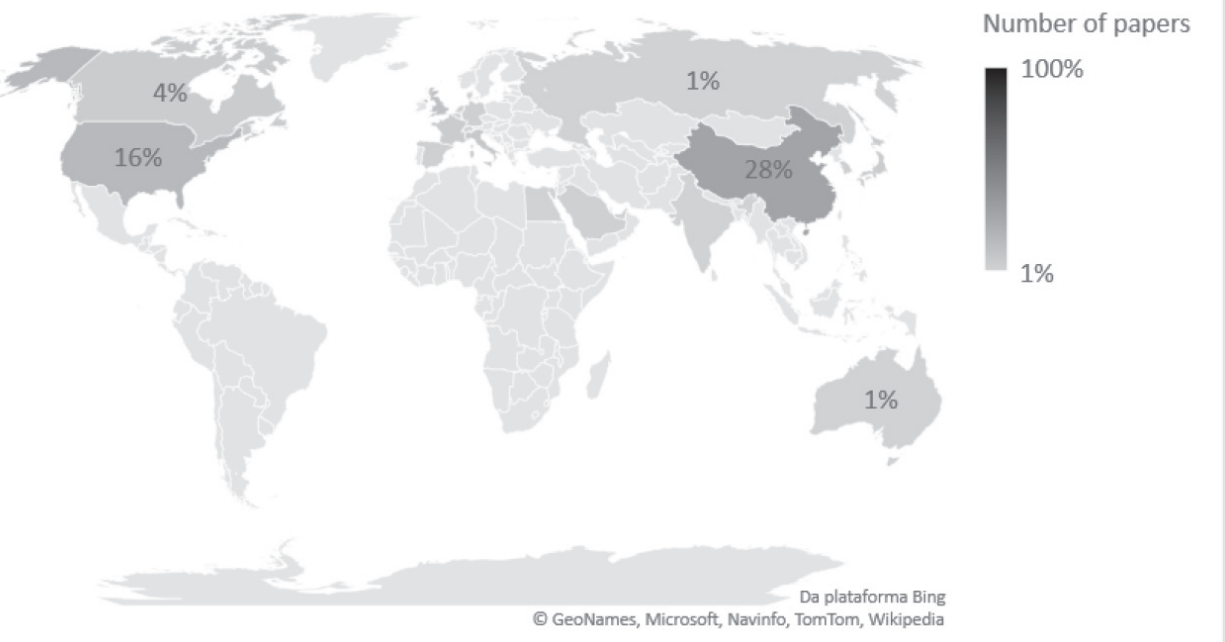

Figure 1. The most cited papers on COVID-19 by country.

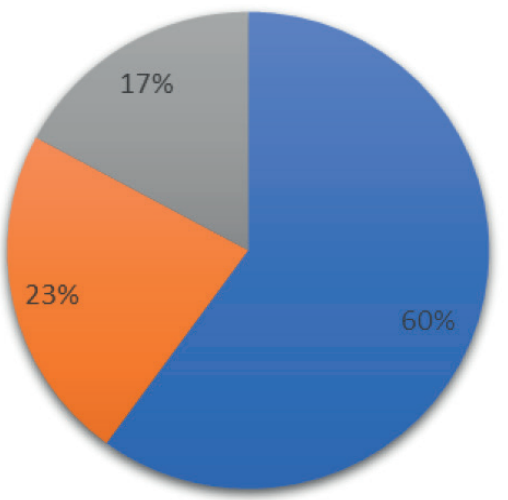

Figure 2. Main approaches on COVID-19 of the most cited papers,

\section{Disease}

COVID-19 was characterized as flu, capable of generating complications in the human respiratory system, such as pneumonia. People with comorbidities or old age were more prone to the most severe disease stage, transmitted through direct contact with infected people and the air. The symptomatic period was more extended than that of ordinary $\mathrm{flu}$, and the transmissibility and infectivity were considered more significant than SARS-CoV, with the prediction of many more contaminated and deaths. The research focus reporting the disease has varied over the months. In February 2020, the

\section{Scientific Trajectory}

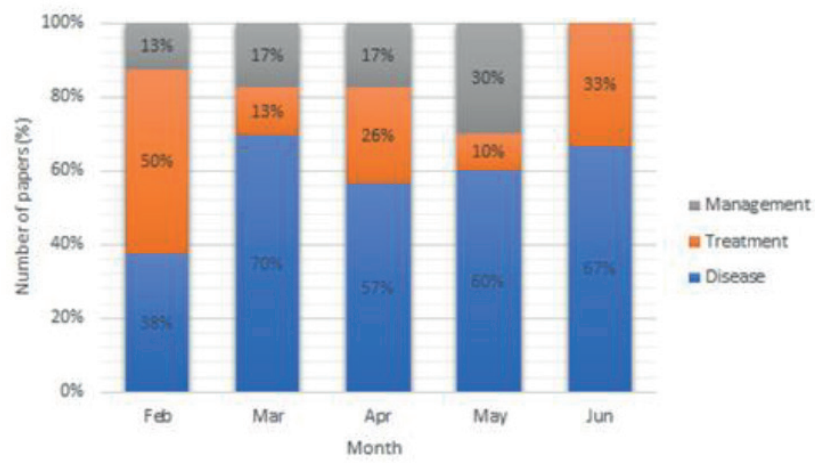

Figure 3. Scientific trajectory on COVID-19 investigations in terms of disease, 107 treatment/prevention and management approaches. concern was to predict the disease's behavior, while in June 2020, it was to identify the health complications caused by COVID-19. The other disease aspects, such as transmission, time, symptoms, and risk factors, were addressed according to their identification and occurrence in the infected people (Figure 4).

\section{Transmission}

Two types of transmission have been identified in scientific research: secondary and tertiary transmissions. In both cases, the transmission occurred through direct contact by droplets exchanged between infected and healthy people, as verified by previous studies ${ }^{6}$. The secondary transmission was also attributed to hospital transmission and contact between asymptomatic and healthy patients. However, the COVID-19 expansion in the world has been attributed to international travel, especially by people from China ${ }^{6}$. One of China's first transmission cases occurred with a 54-year-old Korean man who lived in Wuhan, China. Upon returning to Korea, the man transmitted the disease to a friend (secondary route), who, in turn, transmitted it to his wife, son, and another friend (tertiary route $)^{8}$. At the beginning of the pandemic, both transmission types were also reported in scientific articles; however, in March 2020, the only secondary transmission was reported. In the following months until June 2020, the novel coronavirus's transmission type was not reported by the most cited papers (Figure 5A). The closure of borders and international airports possibly contributed to the reduction of tertiary transmissions in the world.

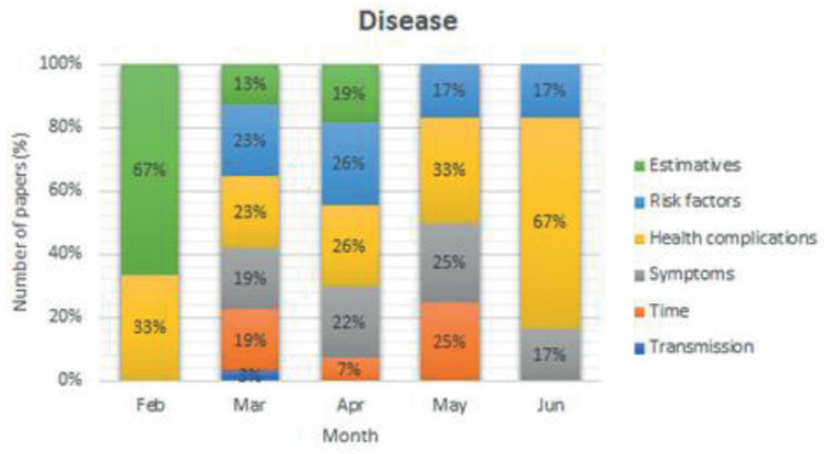

Figure 4. Main disease aspects identified in research on $\mathrm{CO}$ VID-19. 


\section{Time}

The novel coronavirus' behavior and the disease manifestation were correlated with the incubation, communicable, and symptomatic periods, and viral shedding (Figure. 5B). The average incubation period was 5 days ${ }^{4}$, similar to the SARS time, within the expected range for MERS (between 5 and 7 days), and less than the non-SARS (3 days) $)^{9}$. However, other studies have reported an average time between 5 and 7 days, such as 5.8 days $^{10}, 6.4$ days $^{6,11}$, and 6.7 days $^{12}$. The incubation period determination contributes to the monitoring, surveillance, control, and estimation of COVID-199. The incubation period was investigated between March and May 2020, in at least 67\% of the papers, possibly because it represents an important control factor for disease transmission.

The communicable period corresponds to the interval between the positive clinical diagnosis and the negative tests for COVID-19. The average communicable period was 9.5 days, reaching up to 21 days for asymptomatic cases ${ }^{13}$. The average COVID-19 symptoms period was 11.5 days in $97.5 \%$ of patients with an interval of 1.2 days between the onset of symptoms and hospitalization. Disease development was also expected after 14 days of active monitoring or quarantine in about 101 cases for every $10,000^{9}$. The viral shedding median was 20 days, reaching up 37 days in cured patients. The viral shedding has also been identified in killed patients. For the treated patients with antivirals, in the severe and in the critical stages, the viral shedding median was 22,19 , and 24 days, respectively ${ }^{14}$. These four-time parameters were mostly researched between March and May 2020, at least 17\% of published studies.

\section{Symptoms}

The main COVID-19 symptoms were fever, cough, sputum, chills, fatigue, shortness of breath, headache, and olfactory and gustatory disorders. Several asymptomatic cases have also been identified worldwide (Figure 5C). Fever was the most common symptom among patients, and the first identified early in the pandemic ${ }^{15}$, followed by dry cough ${ }^{6}$, fatigue or myalgia, and headache ${ }^{12,16}$. The onset of fever and cough occurred, respectively, in 5 and 7 days $^{8}$, being the fever classified as mild $\left(37.3-38.9^{\circ} \mathrm{C}\right)$ or moderate $\left(38.1-39^{\circ} \mathrm{C}\right)^{16}$. The onset of fatigue occurred in 8 days ${ }^{17}$.

Other symptoms also verified in COVID-19 patients were sputum ${ }^{18}$, chills, shortness of breath or dyspnea ${ }^{12,17}$ diarrhea, and olfactory and gustatory disorders ${ }^{19}$. In almost $86 \%$ of patients, olfactory dysfunctions were identified while gustatory dysfunctions in $88 \%$ of those evaluated in a European clinical study on these symptoms. Both dysfunctions were not associated with nasal obstruction, and the women were more affected than men ${ }^{19}$

Asymptomatic cases were also identified. In a study, 24 asymptomatic cases were evaluated. Approximately 21\% developed symptoms, such as fever, cough, and fatigue during hospitalization, and at least $21 \%$ had complications in the lungs $\mathrm{s}^{13}$. In another study, the COVID- 19 proportion of asymptomatic cases reached $32 \%$, higher than measles (8\%), and lower than polio $(90-95 \%)^{11}$. The information on asymptomatic cases can help COVID-19 infection control and prevent a second outbreak.

\section{Health complications}

COVID-19 is an acute respiratory syndrome that can progress to pneumonia. Clinical evidence of this complication was performed by chest computed tomography (C.T.), which identified bilateral abnormalities, peripheral (showing air bronchograms), ill-defined, and ground-glass opacification ${ }^{20,21}$. These abnormalities predominantly involved the lower lobes of the right lung ${ }^{16,22}$. The multifocal peripheral ground-glass image pattern and mixed opacity prevalent in the lower lung can be seen in the first week of the disease, although many infected people do not have these complications previously ${ }^{18}$ Furthermore, patients may have multiple lobe involvement, interlobular septal thickening ${ }^{23}$, unilobar inverted halo ${ }^{24}$ traction bronchiectasis, and architectural distortion ${ }^{25}$. The diagnosed lesions included irregular lesions, large confluent, and small nodular lesions ${ }^{24}$, being the diffuse or irregular shape, with ground-glass opacity consolidation, observed between 1 and 3 weeks from disease onset ${ }^{18,22}$. Irregular lesions were identified mainly in the lower lobes and along the pleura, while nodular lesions were distributed in the white-vascular bundle forms ${ }^{24}$. These anomalies have also been identified in asymptomatic patients, thus being additional evidence to the laboratory results for an early COVID-19 diagnosis ${ }^{22}$. Chest CT showed a low rate of COVID-19 misdiagnosis, being, therefore, an option for rapid diagnosis of the disease, although it is limited in the identification of the virus type responsible for pulmonary complications ${ }^{26}$. Only in May 2020, at least $80 \%$ of papers reported studies about other pulmonary complications caused by COVID-19 (Figure 5D).

The COVID-19 patients have also shown a decrease in blood oxygen saturation (hypoxemia), a reduced white blood cell count (leukopenia), with a specific reduction in lymphocytes (lymphopenia), and changes in C-reactive protein ${ }^{18}$. Many patients had higher levels of Pt, APTT (activated partial thromboplastin time), d-dimer, lactate dehydrogenase, PCT (procalcitonin), ALB (albumin), and aspartate aminotransferase ${ }^{14,25}$. Patients with previous lung tumors showed edema, exudate, focal reactive pneumocyte hyperplasia with irregular inflammatory cell infiltration, and multinucleated giant cells in the initial phase of COVID-19, even though hyaline membranes were not prominent ${ }^{28}$.

Psychosomatic issues, such as anxiety, stress, and depression during the COVID-19 pandemic, were observed, especially in March and April 2020, in at least 10\% of the most cited papers. An online survey with more than 1,200 people was conducted in 194 Chinese cities. More than half of the interviewees reported moderate to severe psychological impact, $16.5 \%$ depressive symptoms, approximately 29\% anxiety, and just over $8 \%$ some stress. The survey also found that just over $75 \%$ of respondents had concerns about family members' contamination. The most severe levels of mental health complications have been identified among women, especially students ${ }^{29}$

\section{Risk factors}

The main risk factors for COVID-19 involved age, gender, and comorbidities such as hypertension, diabetes, heart disease, and cancer (Figure 5E). Age was considered the leading risk factor by the scientific community since the advanced age increased hospital deaths by a ratio of 1:1014. The average age was 63-64 years, for the most severe cases $^{15,30}$. China and the USA have seen more severe cases in people over the age of $65^{5}$. The children with an average age of 8 years presented mild to moderate symptoms or were diagnosed as asymptomatic in most cases ${ }^{31}$. When associated with the male gender, underlying comorbidities, and progressive radiographic deterioration ${ }^{13,22}$, the death probability was even more remarkable. Hypertension, diabetes, 
and coronary heart disease were identified as comorbidities critical to COVID-1914,30. Obesity was also verified ${ }^{32}$. Patients with acute kidney injury (AKI) had a higher risk of hospital death $^{33}$. Cancer was also assessed as an essential risk factor, whose clinical management requires structure, preparation, and agility on the oncology community ${ }^{34}$, primarily due to the immunocompromise of patients undergoing invasive cancer treatment ${ }^{35}$.

\section{Estimative}

Some scientific studies estimated the number of cases, mortality rate, and contact tracing (especially the zero case) since the beginning of the COVID-19 pandemic (Figure 5F). The increase of cases worldwide was related to international travel and late social isolation established by countries, leading to an immediate outbreak of COVID-19, as previously seen in Italy and Spain, and after in the USA and Brazil. The mortality rates in China and Italy became identical and equal to 2.3\%, with older adults' predominance. The failure to identify the Italian zero case may have influenced that rate ${ }^{36}$. Travel ban measures in Wuhan (China) contributed to the reduction of about $80 \%$ of predicted imported cases in mid-February 2020 , despite a predicted disease progression delay of only 3
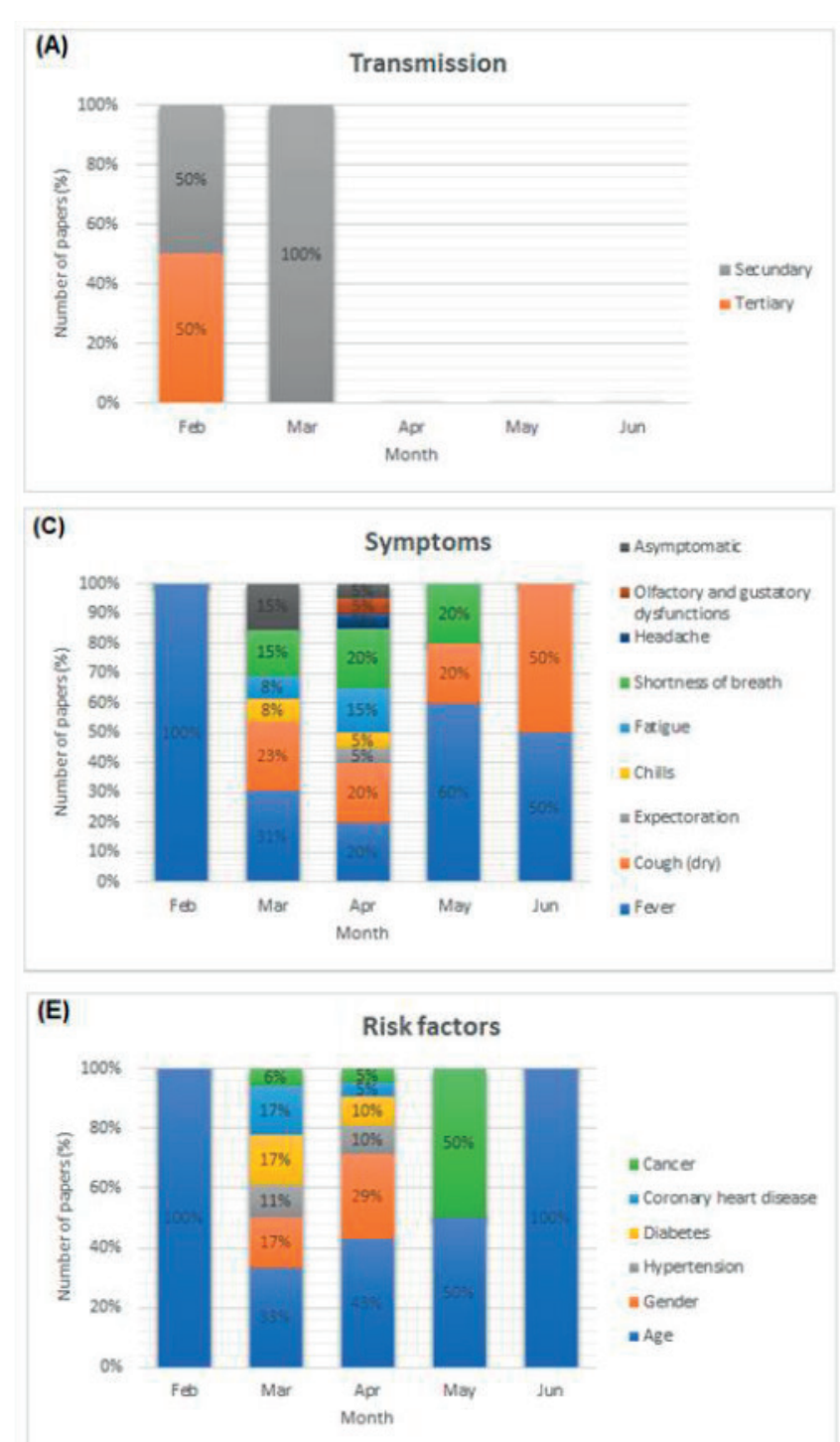

to 5 days. Travel restrictions would only effectively alter the COVID- 19 progress if combined with a reduction of at least $50 \%$ in the community transmissions ${ }^{37}$. Travel restrictions were also beneficial in reducing the average number of daily reproductions in Wuhan, which dropped from 2.35 to 1.05 a week after adopting these measures ${ }^{38}$. All estimates were made using the exponential growth rate from Chinese exported cases for COVID-19, where the necessary number of reproduction (secondary transmission) and the mortality rate could be determined. The COVID-19 pandemic was predicted from two scenarios: from a single case and using the growth rate adjusted with other parameters. Mortality rates ranged from $5.3 \%$ to $8.4 \%$, while the number of secondary cases ranged from 2.1 to 3.2 for the scenarios, respectively ${ }^{39}$. In May and June 2020, research on the COVID-19 cases' estimation was not among the most cited papers in the period, possibly due to disease stabilization in many countries and the interest in other more critical disease aspects.

\section{Treatment and Prevention}

COVID-19 treatments have included strategies to control the disease's leading symptoms and health complications due to the limitations in the rapid development of a vaccine against the
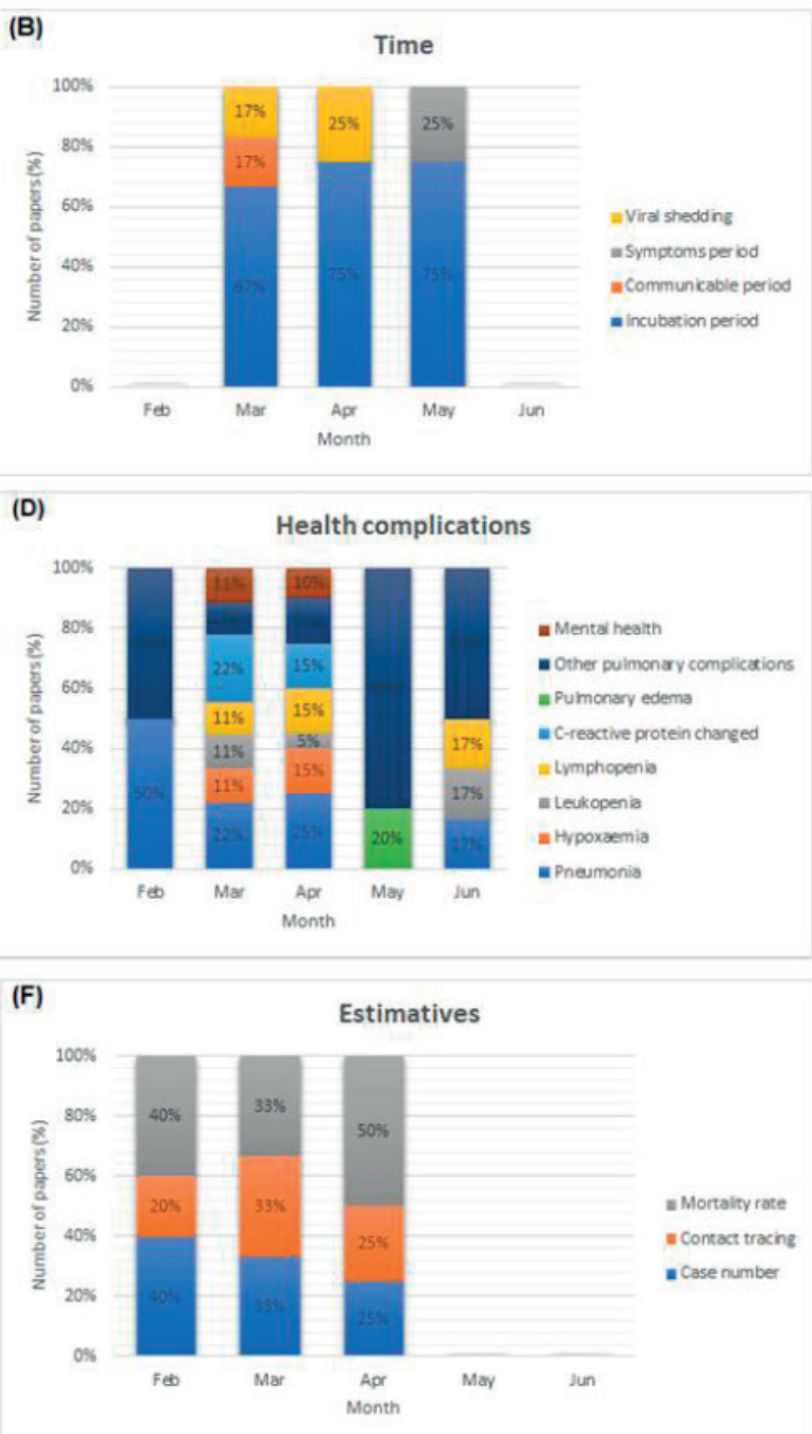

Figure 5. Scientific trajectory on COVID-19 disease in terms of (A) Transmission. (B) Time. (C) Symptoms. (D) Health complications. (E) Risk factors. (F) Estimative. 
SARS-CoV-2. Therefore, different drugs have been investigated since February 2020 as treatment measures, considered palliative and not preventive ones. Medicaments used to fight other viruses have been widely carried out. Alternatively, antibody transfusion and stem cell transplantation have been evaluated in some studies (Figure 6).

\section{Treatment/Prevention}

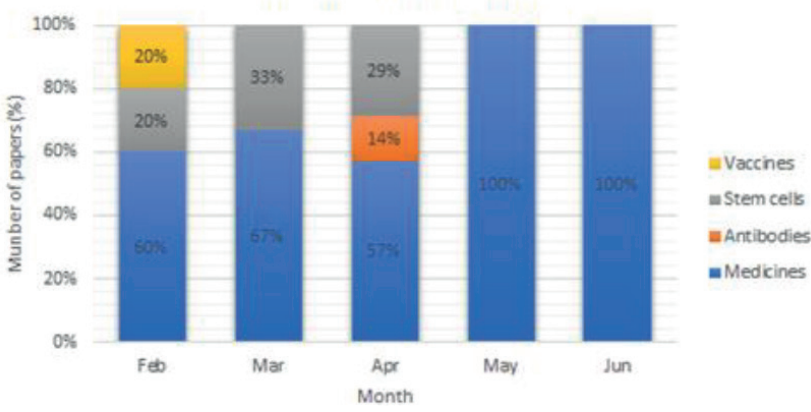

Figure 6. Main treatment and prevention approaches identified in research on COVID-19.

\section{Medicines}

Several antiviral drugs have been investigated (Figure 7). Lopinavir-ritonavir was used to treat HIV (human immunodeficiency virus) and was used to treat COVID-19 since it had previous results in vitro and clinical studies against SARS-CoV. It was prescribed twice a day for 14 days to infected adult patients, and the results were compared to the standard COVID-19 treatment. There was no improvement in patients who used lopinavir-ritonavir ${ }^{40}$. However, the first case of COVID-19 in Korea was treated with lopinavir-ritonavir, which significantly reduced the viral $\beta$-coronavirus loads ${ }^{8}$.

Nucleotide inhibitors such as Anti-HCV used to fight hepatitis $C$ by inhibiting RNA polymerase ${ }^{41}$, and inhibitors of the Renin-Angiotensin-Aldosterone System (RAAS), considered vasoactive peptides ${ }^{42}$ were also investigated. Examples of anti-polymerase drugs with positive results for the COVID-19 treatment were sofosbuvir, IDX-184, ribavirin, and Remdesivir ${ }^{41,43}$. The RAAS inhibitors have been indicated in severe clinical cases, especially in patients with comorbidities related to heart disease. However, the real activity of these inhibitors in combating COVID-19 has been researched. The angiotensin-converting enzyme 2 (ACE2) is considered responsible for coronavirus infection since it functions as a receptor. Studies suggested that RAAS inhibition favors ACE2 overexpression, causing viral activity to increase ${ }^{42}$. Therefore, further studies involving RAAS inhibitors must be carried out to verify their real benefits against COVID- 19 .

Other essential drugs investigated were chloroquine and hydroxychloroquine, which have generated much discussion in the scientific community, although about 20 clinical studies have been carried out in several Chinese hospitals with different coronaviruses SARS-CoV and SAR- CoV-2. These drugs have been used effectively to combat malaria, Q fever, and Whipple's disease, and in a recent study, the chloroquine helped reduce pneumonia, duration of symptoms, and viral shedding of COVID-19, without causing severe side effects ${ }^{44}$. Investigations of drugs such as methylprednisolone, moxifloxacin, interferon alfa-2b physicochemical inhalation, meropenem ${ }^{17,45}$, Kaletra, corticoids ${ }^{16}$, Arbidol and IFN- $\mathbf{a}^{46}$, and the COVID-19 therapies ${ }^{16}$ have also been reported.

\section{Vaccines}

According to $\mathrm{WHO}$, it will take at least 18 months for a vaccine against the novel coronavirus to be completed,

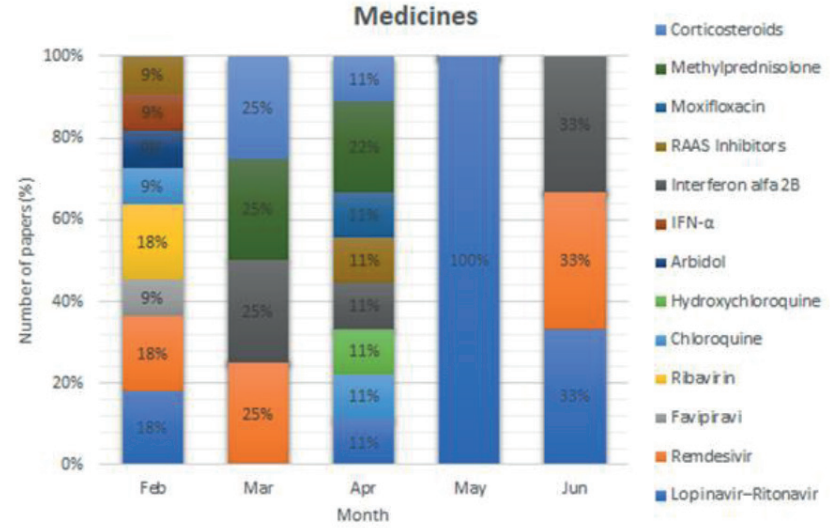

Figure 7. Scientific trajectory on COVID-19 treatment in terms of medicines.

although different research groups and experts are in a real scientific race for its fast development ${ }^{47}$. Currently, treatments only combat symptoms, acting as anti-inflammatory and antiviral drugs. Experience with immunological studies of SARS-CoV and its similarity to SARS-CoV-2 allowed the tracking and identification of $B$ and $T$ cell epitopes in SARSCoV immunogenic structural proteins, also identified in SARSCoV-2. As no epitope mutations were observed in more than 120 SARS-CoV-2 sequences, they were considered useful in researching a new vaccine, with complete and global coverage ${ }^{48}$. Other vaccines have also been investigated, but not reported in the most cited articles in the first half of 2020, possibly because they are confidential research or in little advanced development stages.

\section{Antibodies and Stem Cells}

Alternative COVID-19 treatments have included the convalescent plasma (C.P.) transfusion from recently cured donors containing neutralizing antibodies ${ }^{23}$, and the mesenchymal stem cells (CTMs) transplantation ${ }^{49}$. C.P. therapy was considered efficient in severe COVID-19 cases, contributing to the viremia disappearance in 7 days after the transfusion. An increase in the lymphocyte number and a reduction in C-reactive protein was seen within days, and the lung injuries were absorbed within 7 days $^{23}$. C.P. therapy also helped stabilize body temperature and remove mechanical ventilators, which happened 3 weeks after the transfusion ${ }^{50}$. In turn, the transplantation of MSCs had an immunomodulatory function, which was responsible for an improvement in the pulmonary functions of the patients 2 days after the transplant, a hospital discharge after 10 days, and a general clinical improvement after 14 days. The MSCs transplantation contributed to increased lymphocytes, decreased C-reactive protein, and the disappearance of immune cells secreting overactive cytokines ${ }^{50}$. Although a limited number of people have undergone both treatments, they are promising in combating the novel coronavirus and potential interest in future investigations.

\section{Management of COVID-19}

The management of COVID-19 involved control strategies that range from outstanding medical care and personal hygiene to the social isolation measures. Furthermore, the clinical case determination and the disease importation control, either between countries or between regions of the same country, have been necessary measures for the effective control of COVID-19 (Figure 8). Although these strategies have been prioritized differently from February to June 2020, 


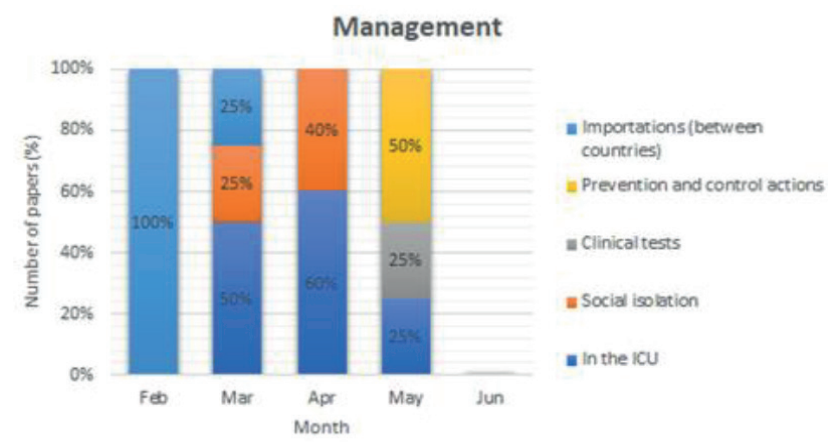

Figure 8. Main management approaches identified in research on COVID-19.

they have all been equally important in reducing the number of cases or stabilizing the COVID-19 and overcoming it in the coming months.

\section{In the Intensive Care Unit (ICU)}

The care for patients admitted to the ICU has involved adopting special medical measures and protocols, which could ensure health professionals' safety and patients' well-being. The main procedures included the infection control (risks of SARS-CoV-2 transmission), laboratory tests, supportive care (continuous renal replacement - CRRT, invasive mechanical ventilation ${ }^{32}$, extracorporeal membrane oxygenation - ECMO ${ }^{47}$, hemodynamic support ${ }^{3}$ ), and COVID-19 therapy ${ }^{3}$. Some procedures were considered specific and necessary in patients with COVID-19, such as emergency tracheal intubation, predicted or unexpected difficult tracheal intubation, cardiac arrest, anesthetic care, and tracheal extubating ${ }^{51}$ (Figure 9A) Although ventilatory support was mostly reported in April 2020 in 56\% of the most cited papers, all measures related to care in the ICU were used equally in May 2020, possibly due to the disease's peak in many countries in this period.

\section{Social Isolation}

Social isolation was a measure used to contain viruses such as SARS and COVID-19, by reducing the disease peak and flattening the curve over time, to ensure sufficient time for the government to implement public health care measures ${ }^{52}$. The main social isolation measures included the closure of schools and workplaces non-essential (non-food and non-health) and people's reduced movement on the streets ${ }^{52}$. Countries that took a long time to implement quarantine measures suffered the consequences of the accelerated increase in cases of COVID-1953. Although COVID- 19 and SARS differ in terms of transmissibility, infectious period, and disease spread, measures taken to control SARS have helped to control COVID-19, such as syndromic surveillance, immediate isolation of infected patients, and strict application of vertical quarantine (lockdown) ${ }^{4}$. Mathematical models considering social isolation and contact tracing could predict the positive impacts on the new COVID-19 cases, thus contributing to the outbreak control ${ }^{10}$. The early relaxation of social isolation compromised COVID-19 control. Therefore, it is necessary to have a strict contact control between people and a policy willing to implement social distance measures until the disease stabilizes and decreases ${ }^{52}$.

\section{Prevention and Control Procedures}

Other COVID-19 prevention and control measures included personal hygiene care such as handwashing after direct contact with infected people or with the environment, the distancing of wild and farm animals ${ }^{6}$, the use of alcohol $70 \%$, and masks in public places. Cancer and dental medicine patients must be treated using particular protocols to reduce the contamination risk ${ }^{54}$. Access to information is crucial for the correct and conscious procedure implementations to combat the novel coronavirus.

\section{Clinical tests}

The COVID-19 diagnoses were carried out by direct and indirect assays, from the determining nucleic acids, immunohistochemical stains, and image tests involving 18F-FDG PET/CT (Figure. 9B). The clinical test based on viral nucleic acid was the primary technique reported ${ }^{13,28,55}$. Immunohistochemical stains are still under development ${ }^{28}$, and the 18F-FDG PET/CT test was able to identify the presence of COVID-19 in tested positive patients. The 18F-FDG PET/CT teste consists of a hybrid technique between positron emission tomography (PET) and conventional computed tomography (C.T.), using the 18F-Fluordeoxiglicose (FDG), a glucose analog, as a tracer. This technique allows for obtaining metabolic and anatomical information. Tomography images helped identify ground-glass peripheral opacities, pulmonary consolidations in lung lobes, and lung lesions with lymph node involvement in patients with COVID-1956.

\section{Importation}

A critical study on vulnerability to COVID-19 in developing countries, especially in Africa, was carried out. The COVID-19 importation was related to the recipient country's ability to respond to the outbreak. Countries such as Egypt, Algeria, and South Africa presented a higher importation risk of the novel coronavirus, but a high capacity to control the disease. Countries such as Ethiopia, Nigeria, Sudan, Angola, Ghana, Tanzania, and Kenya with moderate risk showed high vulnerability to control the outbreak. The same was verified for the provinces of Guangdong, Fujian, and Beijing city ${ }^{57}$. This study can be used to reference countries with limited preparedness to deal with COVID-19, suggesting the adoption of intensified surveillance and resource allocation. Non-African countries can also use these measures.

\section{Conclusions}

It was possible to establish the development level of scientific research on SARS-CoV-2, summarizing the major studies carried out in the first half of 2020, and highlighting the scientific community's main discussions on the disease, treatment/prevention, and management of COVID-19. This compilation study can help future research by identifying knowledge gaps in coronavirus and its disease. Furthermore, the information contained herein may be used in studies on new drugs capable of combating this viral infection, developing vaccines in a shorter period, and on measures to control new COVID-19 cases. This study also helps to understand the disease behavior and proposing solutions for the resumption of economic and social activities by affected countries based on scientific knowledge. All the experience gained with COVID-19 should be used to anticipate future scenarios and establish more effective control procedures to reduce new pandemics' probability in the coming years.

\section{Acknowledgments}

The authors greatly acknowledge the Coordination for the Improvement of Higher Education Personnel (CAPES), the 
(A)
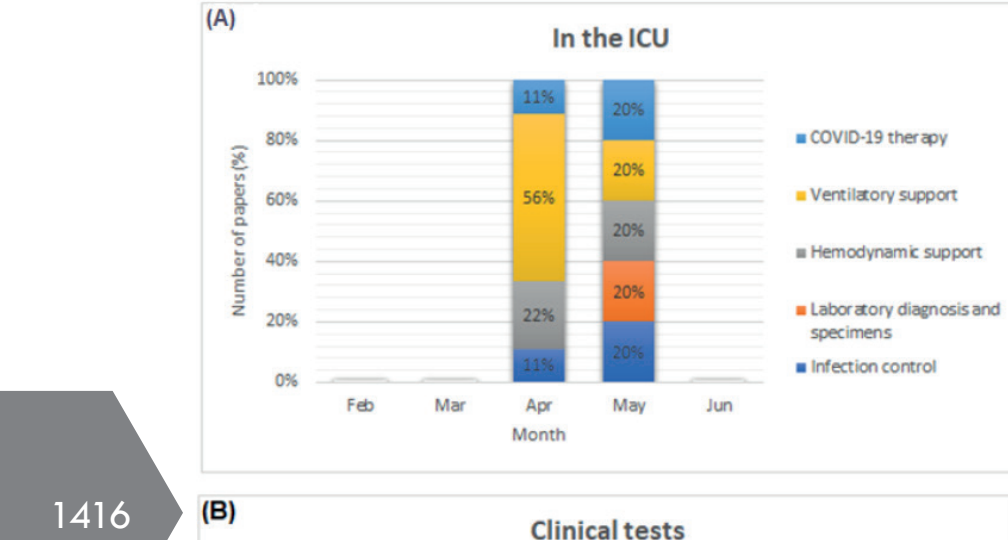

(B)

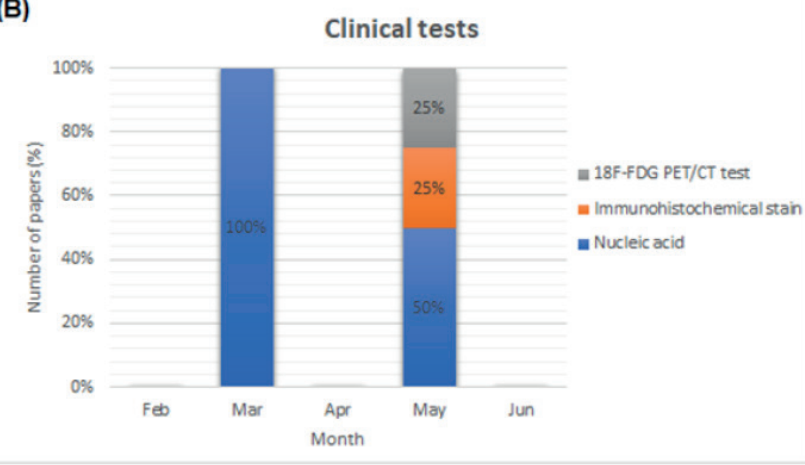

Figure 9. Scientific trajectory on COVID-19 management in terms of (A) Procedures in the ICU. (B) Clinical tests.

National Council for Scientific and Technological Development $(\mathrm{CNPq})$, and the Research Support Foundation of the State of Rio de Janeiro (FAPERJ) for giving financial support for scientific investigation of this paper.

\section{Author contributions}

D.T. and S.B conceived the scientific prospecting project. D.T. searched for the most cited papers on the Web of Science. D.T. and S.B. contributed to the data analysis. D.T. wrote the manuscript, and S.B. revised the text.

\section{Competing interests}

The authors declare that there are no competing interests.

\section{Data and materials availability}

All data are available in the main text or the supplementary materials.

\section{Ethics statement}

The authors confirm that the ethical policies of the journal, as noted on the journal's author guidelines page, have been adhered to. No ethical approval was required as this is a review article with no original research data.

\section{Bibliographic references}

1. Pan American Health Organization. Coronavirus Disease (COVID-19) pandemic [Internet]. [cited 2020 Jul 2]. Available from: https://www.paho.org/en

2. Wordl Health Organization. Coronavirus disease (COVID-19) pandemic [Internet]. [cited 2020 Jul 2]. Available from: https://www. who.int/emergencies/diseases/novel-coronavirus-2019
3. Alhazzani W, Møller MH, Arabi YM, Loeb M, Gong MN, Fan E, et al. Surviving Sepsis Campaign: guidelines on the management of critically ill adults with Coronavirus Disease 2019 (COVID-19) [Internet]. Vol. 46, Intensive Care Medicine. Springer Berlin Heidelberg; 2020. 854-887 p. Available from: https://doi.org/10.1007/ s00134-020-06022-5

4. Wilder-Smith A, Chiew CJ, Lee VJ. Can we contain the COVID-19 outbreak with the same measures as for SARS? Lancet Infect Dis [Internet]. 2020;20(5):e102-7. Available from: http://dx.doi. org/10.1016/S1473-3099(20)30129-8

5. Bialek S, Boundy E, Bowen V, Chow N, Cohn A, Dowling N, et al. Severe outcomes among patients with coronavirus disease 2019 (COVID-19) - United States, February 12-march 16, 2020. Morb Mortal Wkly Rep. 2020;69(12):343-6.

6. Lai CC, Shih TP, Ko WC, Tang HJ, Hsueh PR. Severe acute respiratory syndrome coronavirus 2 (SARS-CoV-2) and coronavirus disease-2019 (COVID-19): The epidemic and the challenges. Int $J$ Antimicrob Agents [Internet]. 2020;55(3):105924. Available from: https://doi.org/10.1016/j.ijantimicag.2020.105924

7. Zhang T, Wu Q, Zhang Z. Probable Pangolin Origin of SARSCoV-2 Associated with the COVID-19 Outbreak. Curr Biol [Internet]. 2020;30(7):1346-1351.e2. Available from: https://doi. org/10.1016/j.cub.2020.03.022

8. Lim J, Jeon S, Shin HY, Kim MJ, Seong YM, Lee WJ, et al. The Author's response: Case of the index patient who caused tertiary transmission of coronavirus disease 2019 in Korea: The Application of lopinavir/ritonavir for the treatment of COVID-19 pneumonia monitored by quantitative RT-PCR. J Korean Med Sci. 2020;35(7):1-6.

9. Lauer SA, Grantz KH, Bi Q, Jones FK, Zheng Q, Meredith HR, et al. The incubation period of coronavirus disease 2019 (CoVID-19) from publicly reported confirmed cases: Estimation and application. Ann Intern Med. 2020;172(9):577-82.

10. Hellewell J, Abbott S, Gimma A, Bosse NI, Jarvis Cl, Russell TW, et al. Feasibility of controlling COVID-19 outbreaks by isolation of cases and contacts. Lancet Glob Heal. 2020;8(4):e488-96.

11. Mizumoto K, Kagaya K, Zarebski A, Chowell G. Estimating the asymptomatic proportion of coronavirus disease 2019 (COVID-19) cases on board the Diamond Princess cruise ship, Yokohama, Japan, 2020. Eurosurveillance. 2020;25(10):1-5.

12. Tian S, Hu N, Lou J, Chen K, Kang X, Xiang Z, et al. Characteristics of COVID-19 infection in Beijing. J Infect [Internet]. 2020;80(4):401-6. Available from: https://doi.org/10.1016/j.jinf.2020.02.018

13. Hu Z, Song C, Xu C, Jin G, Chen Y, Xu X, et al. Clinical characteristics of 24 asymptomatic infections with COVID-19 screened among close contacts in Nanjing, China. Sci China Life Sci. 2020;63(5):706-11.

14.Zhou F, Yu T, Du R, Fan G, Liu Y, Liu Z, et al. Clinical course and risk factors for mortality of adult inpatients with COVID-19 in Wuhan, China: a retrospective cohort study. Lancet [Internet]. 2020;395(10229):1054-62. Available from: http://dx.doi. org/10.1016/S0140-6736(20)30566-3

15. Bhatraju PK, Ghassemieh BJ, Nichols M, Kim R, Jerome KR, Nalla AK, et al. COVID-19 in critically ill patients in the Seattle region Case series. N Engl J Med. 2020;382(21):2012-22.

16. Wan S, Xiang Y, Fang W, Zheng Y, Li B, Hu Y, et al. Clinical features and treatment of COVID-19 patients in northeast Chongqing. $J$ Med Virol. 2020;(March):1-10.

17. Xu Z, Shi L, Wang Y, Zhang J, Huang L, Zhang C, et al. Pathological findings of COVID-19 associated with acute respiratory distress syndrome. Lancet Respir Med [Internet]. 2020;8(4):420-2. Available from: http://dx.doi.org/10.1016/S2213-2600(20)30076-X

18. Yang W, Cao Q, Qin L, Wang X, Cheng Z, Pan A, et al. Clinical characteristics and imaging manifestations of the 2019 novel coronavirus disease (COVID-19):A multi-center study in Wenzhou city, Zhejiang, China. J Infect [Internet]. 2020;80(4):388-93. Available from: https://doi.org/10.1016/j.jinf.2020.02.016 
19. Lechien JR, Chiesa-Estomba CM, De Siati DR, Horoi M, Le Bon SD, Rodriguez A, et al. Olfactory and gustatory dysfunctions as a clinical presentation of mild-to-moderate forms of the coronavirus disease (COVID-19): a multicenter European study. Eur Arch Oto-Rhino-Laryngology [Internet]. 2020;2(0123456789). Available from: https:// doi.org/10.1007/s00405-020-05965-1

20. Bernheim A, Mei X, Huang M, Yang Y, Fayad ZA, Zhang N, et al. Chest CT findings in coronavirus disease 2019 (COVID-19): Relationship to duration of infection. Radiology. 2020;295(3):685-91.

21. Pan F, Ye T, Sun P, Gui S, Liang B, Li L, et al. Time course of lung changes at chest C.T. during recovery from Coronavirus disease 2019 (COVID-19). Radiology. 2020;295(3):715-21.

22. Shi H, Han X, Jiang N, Cao Y, Alwalid O, Gu J, et al. Radiological findings from 81 patients with COVID-19 pneumonia in Wuhan, China: a descriptive study. Lancet Infect Dis [Internet]. 2020;20(4):425-34 Available from: http://dx.doi.org/10.1016/S1473-3099(20)30086-4

23. Duan K, Liu B, Li C, Zhang H, Yu T, Qu J, et al. Effectiveness of convalescent plasma therapy in severe COVID-19 patients. Proc Natl Acad Sci U S A. 2020:117(17):9490-6.

24. Yoon SH, Lee KH, Kim JY, Lee YK, Ko H, Kim KH, et al. chest radiographic and ct findings of the 2019 novel coronavirus disease (Covid-19): Analysis of nine patients treated in korea. Korean J Radiol. 2020;21(4):498-504.

25. Zhao W, Zhong Z, Xie X, Yu Q, Liu J. Relation between chest C.T. findings and clinical conditions of coronavirus disease (covid-19) pneumonia: A multicenter study. Am J Roentgenol. 2020;214(5):1072-7.

26. Li Y, Xia L. Coronavirus disease 2019 (COVID-19): Role of chest C.T. in diagnosis and management. Am J Roentgenol. 2020;214(6):1280-6.

27. Thachil J, Tang N, Gando S, Falanga A, Cattaneo M, Levi M, et al. ISTH interim guidance on recognition and management of coagulopathy in COVID-19. J Thromb Haemost. 2020;(March):1023-6.

28. Tian S, Hu W, Niu L, Liu H, Xu H, Xiao SY. Pulmonary Pathology of Early-Phase 2019 Novel Coronavirus (COVID-19) Pneumonia in Two Patients With Lung Cancer. J Thorac Oncol [Internet]. 2020;15(5):7004. Available from: https://doi.org/10.1016/j.jtho.2020.02.010

29. Wang C, Pan R, Wan X, Tan Y, Xu L, Ho C.S., et al. Immediate psychological responses and associated factors during the initial stage of the 2019 coronavirus disease (COVID-19) epidemic among the general population in China. Int J Environ Res Public Health. 2020;17(5)

30. Wu J, Li W, Shi X, Chen Z, Jiang B, Liu J, et al. Early antiviral treatment contributes to alleviate the severity and improve the prognosis of patients with novel coronavirus disease (COVID-19). J Intern Med. 2020;2:1-11

31. Qiu H, Wu J, Hong L, Luo Y, Song Q, Chen D. Clinical and epidemiological features of 36 children with coronavirus disease 2019 (COVID-19) in Zhejiang, China: an observational cohort study. Lancet Infect Dis [Internet]. 2020;20(6):689-96. Available from: http:// dx.doi.org/10.1016/S1473-3099(20)30198-5

32. Richardson S, Hirsch JS, Narasimhan M, Crawford JM, McGinn T, Davidson KW, et al. Presenting Characteristics, Comorbidities, and Outcomes among 5700 Patients Hospitalized with COVID-19 in the New York City Area. JAMA - J Am Med Assoc. 2020;323(20):2052-9.

33. Cheng Y, Luo R, Wang K, Zhang M, Wang Z, Dong L, et al. Kidney disease is associated with in-hospital death of patients with COVID-19. Kidney Int [Internet]. 2020;97(5):829-38. Available from: https://doi. org/10.1016/j.kint.2020.03.005

34. Ueda M, Martins R, Hendrie PC, McDonnell T, Crews JR, Wong TL, et al. Managing Cancer Care During the COVID-19 Pandemic: Agility and Collaboration Toward a Common Goal. J Natl Compr Canc Netw. 2020:18(4):1-4

35. Al囚Shamsi HO, Alhazzani W, Alhuraiji A, Coomes EA, Chemaly RF, Almuhanna M, et al. A Practical Approach to the Management of Cancer Patients During the Novel Coronavirus Disease 2019 ( COVID 囚19) Pandemic: An International Collaborative Group . Oncologist. 2020;2019:1-10

36. Porcheddu R, Serra C, Kelvin D, Kelvin N, Rubino S. Similarity in Case Fatality Rates (CFR) of COVID-19/SARS-COV-2 in Italy and China. J Infect Dev Ctries. 2020;14(2):125-8.
37. Chinazzi M, Davis JT, Ajelli M, Gioannini C, Litvinova M, Merler S, et al. The effect of travel restrictions on the spread of the 2019 novel coronavirus (COVID-19) outbreak. Science (80- ). 2020;368(6489):395-400.

38. Kucharski AJ, Russell TW, Diamond C, Liu Y, Edmunds J, Funk S, et al. Early dynamics of transmission and control of COVID-19: a mathematical modelling study. Lancet Infect Dis. 2020;20(5):553-8.

39. Jung S, Akhmetzhanov AR, Hayashi K, Linton NM, Yang Y, Yuan B, et al. Real-Time Estimation of the Risk of Death from Novel Coronavirus (COVID-19) Infection: Inference Using Exported Cases. J Clin Med. 2020;9(2):523

40. Cao B, Wang Y, Wen D, Liu W, Wang J, Fan G, et al. A trial of lopinavir-ritonavir in adults hospitalized with severe covid-19. N Engl J Med. 2020:382(19):1787-99.

41. Elfiky AA. Anti-HCV, nucleotide inhibitors, repurposing against COVID-19. Life Sci. 2020;248(January).

42. Vaduganathan M, Vardeny O, Michel T, McMurray JJV, Pfeffer MA, Solomon SD. Renin-Angiotensin-Aldosterone System Inhibitors in Patients with Covid-19. N Engl J Med [Internet]. 2020 Apr 23;382(17):1653-9. Available from: http://www.nejm.org/doi/10.1056/NEJMsr2005760

43. Grein J, Ohmagari N, Shin D, Diaz G, Asperges E, Castagna A, et al. Compassionate use of remdesivir for patients with severe Covid-19. N Engl J Med. 2020;382(24):2327-36.

44. Colson P, Rolain JM, Lagier JC, Brouqui P. Raoult D. Chloroquine and hydroxychloroquine as available weapons to Fight COVID-19. Int J Antimicrob Agents [Internet]. 2020;55(4):105932. Available from: https:// doi.org/10.1016/j.jijantimicag.2020.105932

45. Zhu L, Xu X, Ma K, Yang J, Guan H, Chen S, et al. Successful recovery of COVID-19 pneumonia in a renal transplant recipient with long-term immunosuppression. Am J Transplant. 2020;(March):1-5.

46. Dong L, Hu S, Gao J. Discovering drugs to treat coronavirus disease 2019 (COVID-19). Drug Discov Ther. 2020;14(1):58-60.

47. Jiang F, Deng L, Zhang L, Cai Y, Cheung CW, Xia Z. Review of the Clinical Characteristics of Coronavirus Disease 2019 (COVID-19). J Gen Intern Med. 2020;35(5):1545-9.

48. Ahmed SF, Quadeer AA, McKay MR. Preliminary identification of potential vaccine targets for the COVID-19 Coronavirus (SARS-CoV-2) Based on SARS-CoV Immunological Studies. Viruses. 2020;12(3).

49. Leng Z, Zhu R, Hou W, Feng Y, Yang Y, Han Q, et al. Transplantation of ACE2-Mesenchymal stem cells improves the outcome of patients with covid-19 pneumonia. Aging Dis. 2020;11(2):216-28.

50. Shen C, Wang Z, Zhao F, Yang Y, Li J, Yuan J, et al. Treatment of 5 Critically IIL Patients with COVID-19 with Convalescent Plasma. JAMA - J Am Med Assoc. 2020;323(16):1582-9.

51. Cook TM, El-Boghdadly K, McGuire B, McNarry AF, Patel A, Higgs $A$. Consensus guidelines for managing the airway in patients with COVID-19: Guidelines from the Difficult Airway Society, the Association of Anaesthetists the Intensive Care Society, the Faculty of Intensive Care Medicine and the Royal College of Anaesthetist. Anaesthesia. 2020;785-99.

52. Prem K, Liu Y, Russell TW, Kucharski AJ, Eggo RM, Davies N, et al. The effect of control strategies to reduce social mixing on outcomes of the COVID-19 epidemic in Wuhan, China: a modelling study. Lancet Public Heal. 2020;5(5):e261-70.

53. Remuzzi A, Remuzzi G. COVID-19 and Italy: what next? Lancet [Internet]. 2020;395(10231):1225-8. Available from: http://dx.doi.org/10.1016/ S0140-6736(20)30627-9

54. Meng L, Hua F, Bian Z. Coronavirus Disease 2019 (COVID-19): Emerging and Future Challenges for Dental and Oral Medicine. J Dent Res. 2020;99(5):481-7.

55. Xia W, Shao J, Guo Y, Peng X, Li Z, Hu D. Clinical and C.T. features in pediatric patients with COVID-19 infection: Different points from adults. Pediatr Pulmonol. 2020;55(5):1169-74.

56. Qin C, Liu F, Yen TC, Lan X. 18F-FDG PET/CT findings of COVID-19: a series of four highly suspected cases. Eur J Nucl Med Mol Imaging. 2020;47(5):1281-6.

57. Gilbert M, Pullano G, Pinotti F, Valdano E, Poletto C, Boëlle PY, et al. Preparedness and vulnerability of African countries against importations of COVID-19: a modeling study. Lancet. 2020;395(10227):871-7. 